\title{
Cord Blood Neutrophil Phagocytic Index in Neonates Born to Anemic Mothers and in Neonates Born with Risk of Early-onset Sepsis
}

\author{
Grisilda V Bernhardt ${ }^{1}$, Malay Jhancy ${ }^{2}$, Liegelin Kavitha Bernhardt' ${ }^{3}$, Pooja Shivappa ${ }^{4}$, Suresh Kumar ${ }^{5}$, Janita RT Pinto ${ }^{6}$
}

\begin{abstract}
Introduction: During early life, the neutrophils as components of the innate immune system help to defend against pathogenic infections. Evaluation of cord blood neutrophil phagocytic index (NPI) has considerable value for understanding innate immune status. Few previous studies have investigated the association of maternal iron levels and neonatal immune status. The association of prenatal factors leading to increased risk of early-onset sepsis (EOS) in neonates is well understood. Neutrophils as components of the innate immune system represent the first line of defense against pathogens and are important especially during early life. Compromised neutrophil phagocytic functions and immune responses have been linked to the development of EOS in neonates.

Aims and objectives: (1) To evaluate the phagocytic index of the neutrophils in the cord blood obtained from neonates born to anemic mothers. (2) To evaluate the phagocytic index of the neutrophils in the cord blood obtained from neonates born with risk factors for developing EOS.

Materials and methods: To study NPI in neonates born to anemic mothers, 60 mothers and newborn pairs were recruited and subdivided into anemic and nonanemic groups, based on hemoglobin $(\mathrm{Hb})$ levels of maternal blood. To study NPI in cord blood of neonates born with risk factors for EOS, 61 neonates with two or more risk factors for developing sepsis were recruited, along with 48 healthy infants who served as controls. Venous blood samples were collected 1.5 hours \pm 20 minutes before the delivery. Five milliliters of cord blood was collected soon after childbirth. Results: The mean NPI values and also the mean gestational age and birth weight were significantly lower $(p<0.05)$ in neonates of anemic mothers. Positive linear correlation (inverse relationship) of $r=0.67$ and $p<0.05$ was observed between maternal Hb and NPI. Neonates with risk of EOS had significantly $(p<0.05)$ lower values of NPI and significantly elevated levels of C-reactive proteins (CRP).

Conclusions: There exists a significant association between maternal iron status and lowered phagocytic capacity of neutrophils, suggesting compromised innate immunity in neonates. Lowered NPI in the cord blood of infants born with risk of EOS is suggestive of the compromised immune response, which may add to the risk of developing EOS. Lowered cord blood NPI may mark early neonatal immune deficiency and immune processes in neonates. Further studies on cord blood NPI can lead to identifying NPI as an early predictor of EOS.
\end{abstract}

Keywords: Cord blood, Innate immunity, Maternal anemia, Neonatal sepsis, Neutrophil phagocytic index, Phagocytic capacity.

Journal of South Asian Federation of Obstetrics and Gynaecology (2021): 10.5005/jp-journals-10006-1851a

\section{INTRODUCTION}

Despite advancements in neonatal medicine, the risk of neonatal mortality remains significantly high ${ }^{1,2}$ It is well understood that maternal factors are associated with neonatal morbidity and mortality. ${ }^{3}$ Maternal anemia, premature rupture of membranes (more than 18 hours), meconium-stained amniotic fluid, foul-smelling amniotic fluid, gestational age $<34$ weeks, low birth weight $(<2500 \mathrm{~g})$, gender, sex (male), mode of delivery, preeclampsia, and low Apgar scores are some of the risk factor associated with poor neonatal outcome and are considered as risk factors for early-onset sepsis(EOS) in neonates. ${ }^{3-5}$ Sepsis that develops within the first 72 hours of life is termed as EOS. In clinical practice, neonates born with two or more of these risk factors are carefully screened for EOS., ${ }^{3,5}$ Maternal iron deficiency and other risk factors for sepsis are reported to alter the functions of neutrophils. $3 ., 4,6$

Maternal micronutrient deficiency and more often iron deficiency are matters of concern in most of the developing countries. Maternal anemia is seen to prevail in more than $80 \%$ of pregnant women in developing countries. ${ }^{1,6}$ Maternal iron deficiency anemia is significantly associated with both maternal and fetal adverse outcomes. Preterm labor, low birth weight, preeclampsia, impairment of growth, development, and chronic
${ }^{1}$ Department of Biochemistry, RAK College of Medical Sciences, RAKMedical and Health Science University, Ras Al Khaimah, United Arab Emirates

${ }^{2}$ Department of Paediatrics, RAK College of Medical Sciences, RAK Medical and Health Science University, Ras Al Khaimah, United Arab Emirates

${ }^{3}$ Department of Physiology, Melaka Manipal Medical College, Manipal Campus, Manipal Academy of Higher Education, Manipal, Karnataka, India ${ }^{4}$ Department of Basic Sciences, RAK College of Medical Sciences, RAK Medical and Health Science University, Ras Al Khaimah, United Arab Emirates

${ }^{5}$ Department of Pharmacology, RAK College of Medical Sciences, RAK Medical and Health Science University, Ras Al Khaimah, United Arab Emirates ${ }^{6}$ Department of Biomedical Sciences, College of Medicine, Gulf Medical University, Ajman, United Arab Emirates

Corresponding Author: Janita RT Pinto, Department of Biomedical Sciences, College of Medicine, Gulf Medical University, Ajman, United Arab Emirates, Phone: +009710566944722, e-mail: dr.pjanita@gmu.ac.ae

How to cite this article: Bernhardt GV, Jhancy M, Bernhardt LK, et al. Cord Blood Neutrophil Phagocytic Index in Neonates Born to Anemic Mothers and in Neonates Born with Risk of Early-onset Sepsis. J South Asian Feder Obst Gynae 2021;13(1):26-30.

Source of support: Nil

Conflict of interest: None

( ) Jaypee Brothers Medical Publishers. 2021 Open Access This article is distributed under the terms of the Creative Commons Attribution 4.0 International License (https://creativecommons.org/licenses/by-nc/4.0/), which permits unrestricted use, distribution, and non-commercial reproduction in any medium, provided you give appropriate credit to the original author(s) and the source, provide a link to the Creative Commons license, and indicate if changes were made. The Creative Commons Public Domain Dedication waiver (http://creativecommons.org/publicdomain/zero/1.0/) applies to the data made available in this article, unless otherwise stated. 
diseases later in life are some of the consequences of maternal anemia. ${ }^{1,7,8}$

Maternal micronutrient status plays a role in programming the child's immune mechanisms by directly affecting Tlymphocyte and natural cell populations of the infants. ${ }^{6,9}$

Phagocytic neutrophils are important components of the innate immune system and serve as the first line of invaders against pathogens during early life. ${ }^{10-12}$ Chemotactic and phagocytic capacities of neutrophils help them to mediate essential immune functions; firstly, they route ingested pathogens to lysosomes that are abundant in hydrolases, and thereby they initiate a pathway for microbial death. ${ }^{13,14}$ They also direct the antigens to major histocompatibility pathways, thus serving a dual role: as a cellular immune effector and as a bridge between the innate and acquired immune responses. ${ }^{13,15}$ Decreased chemotactic response of these cord blood phagocytes suggests compromised immune response. ${ }^{11,14}$ Neutrophils kill invading bacteria with potent oxygendependent and oxygen-independent microbicidal systems, which are the basis for their phagocytic capacity, ${ }^{16}$ and phagocytic capacity can be studied as neutrophil phagocytic index (NPI). ${ }^{17}$

We found no considerable studies that evaluated cord blood $\mathrm{NPI}$ in neonates born with risk factors for sepsis as markers of immunity.

Evaluation of cord blood NPI has considerable value for understanding innate immune status.

We hypothesized that NPI may be altered in cord blood neutrophils in neonates born with risk of EOS and may serve as a marker for innate immune response.

Thus, in this study, NPI in the cord blood neutrophils isolated from neonates born to anemic mothers and neonates born with two or more risk factors for EOS was analyzed.

\section{Materials and Methods}

The study design was a cross-sectional analysis and was conducted in a diagnostics and research center in South India. The Institutional Ethical Committee approved the protocol (Reference ID: 201201340). The procedures followed were in accordance with the ethical standards of the Institutional Human Ethics Committee and with the Helsinki Declaration. Prior informed consent was obtained from all participating mothers.

- NPI of the neutrophil in the cord blood of infants born to anemic mothers: This was studied in cord blood neutrophils of neonates born to anemic mothers. Sixty mothers of age ranging from 21 to 35 years along with infant pairs were recruited after obtaining informed consent from the mothers. Thirty out of the 60 participants were not anemic and served as age-matched controls.

The enrolled mother-infant pairs were grouped based on maternal hemoglobin levels. Mothers with $\mathrm{Hb}$ less than $11 \mathrm{~g} / \mathrm{dL}$ were considered as anemic (group $1, N=30$ ), and mothers with $\mathrm{Hb}$ more than $11 \mathrm{~g} / \mathrm{dL}$ were considered nonanemic and served as controls (group 2, $N=30$ ).

Inclusion criteria: All pregnant women $\geq 26$ weeks gestation who consented to participate and their newborn pairs were recruited for the study.

Exclusion criteria: Pregnant women who presented with medical problems such as gestational diabetes, hypertension, hypothyroidism, epilepsy, renal diseases, cardiorespiratory diseases, immunodeficiency syndromes, antepartum hemorrhage, history of blood transfusion in antenatal period, babies with major congenital anomalies, pathological jaundice, and twins births were all excluded from the study.

- NPI in the cord blood of neonates born with risk of EOS: To study phagocytic index in cord blood neutrophils of neonates born with risk factors for developing EOS, 61 mothers within the age-group of 21-35 years with their infant pairs were recruited after obtaining informed consent from the mothers (group A). Neonates in group A were those who were born with at least two of known risk factors for developing EOS as described in Tables. Cord blood samples from 48 healthy neonates born to mothers within the age-group of 21-35 years served as controls (group B).

Pregnant women who were severely sick because of medical conditions such as diabetes, renal disease cardiorespiratory disease, chronic hypertension, and chromosomal anomalies were excluded.

To determine $\mathrm{Hb}$ levels, maternal venous blood samples were collected 1.5 hours \pm 20 minutes before the delivery. The hemoglobin concentration was determined by the standard spectrophotometric cyanmethemoglobin method.

To study NPI, $5 \mathrm{~mL}$ of umbilical cord blood was collected by venipuncture of umbilical vein into plastic tubes containing anticoagulant following childbirth, after clamping the cord with two clamps placed 4-5 inches apart.

The phagocytic activity of neutrophils was determined by a standard method. The phagocytic index, i.e., the number of microbial bodies absorbed by an average leukocyte, was evaluated in the isolated neutrophils.

Isolation of neutrophils from cord blood was performed using commercially available Polymorphprep gradient following the manufacturer's instructions.

The neutrophils were resuspended in RPMI 1640 culture medium at a final concentration of $9 \times 10^{5}$ cells $/ \mathrm{mL}$. Trypan blue dye exclusion staining was used to check cell viability.

About $5 \times 10^{8}$ Staphylococcus aureus cells in buffer were centrifuged at $1200 \mathrm{rpm}$, washed with phosphate buffer saline (PBS), and were treated with whole serum and kept at $37^{\circ} \mathrm{C}$ for 45 minutes with shaking for opsonization. Following opsonization, pellets got after centrifugation were washed in PBS and further reconstituted in PBS. Bacteria and neutrophils (1:9) were then processed for phagocytosis by incubating at $37^{\circ} \mathrm{C}$ for 30 minutes. Finally, smears were prepared, stained, and observed under $100 \times$ objective for phagocytized bacteria. The number of bacteria engulfed by 200 neutrophils was counted. The ingestion bacteria was expressed as a phagocytic index, which was a percentage of the total bacteria added in the reaction mixture. ${ }^{17}$

\section{Analysis of the Results}

Values of study parameters are represented as mean and standard deviation. Paired Student's $t$-test was used to compare and analyze the data between group 1 and group 2, and $p$ value of less than 0.05 was considered statistically significant.

Descriptive statistics were used for baseline variables. Correlation between maternal $\mathrm{Hb}$ and cord blood NPI was done by regression analysis, using SPSS software. $r=0.87$ and $p<0.05$ were considered a significant positive linear correlation.

To analyze the association of cord blood NPI in neonates born with at least two risk factors for EOS and control groups, an unpaired $t$-test was used. Independent $t$-test of $p$ value $<0.05$ was considered as statistically significant. The SPSS software package (version 20) was used for analysis. 


\section{Results}

- NPI of the neutrophil in the cord blood of infants born to anemic mothers: Among delivered newborns, 46.6\% $(n=28)$ were males and $53.4 \%(n=32)$ were females. The mean $\mathrm{Hb}$ levels in mothers in group 2 were significantly higher when compared to mean $\mathrm{Hb}$ levels in mothers in group 1.

As presented in Table 1 and Figure 1, NPI was significantly lower $(p<0.05)$ in neutrophils isolated from cord blood of group 1 neonates (neonates born to anemic mothers) when compared to group 2.

Bivariate regression analysis that was done to correlate the relationship between maternal $\mathrm{Hb}$ and cord blood NPI shows a significant positive linear correlation inverse relationship $(r=0.67$, $p<0.05$, between maternal $\mathrm{Hb}$ and cord blood NPI ), indicating a significantly lower value of NPI in neonates born to anemic mothers (Figs 1 and 2).

Additionally, we observed that the mean gestational age was significantly lower in neonates in group $1(28.07 \pm 2.39 \mathrm{~g})$ when compared to gestational age of neonates in group 2 $(37.93 \pm 5.2 \mathrm{~g})$. The mean birth weight of neonates in group 1 was also was significantly lower when compared to the mean birth weight of neonates in group 2.

- NPI in the cord blood of neonates born with risk of EOS: Neonates born with at least two positive risk factors for EOS (group A) showed significantly $(p<0.05)$ lower values of NPI, in their cord blood neutrophils when compared with the $\mathrm{NPI}$ values of neonates in group B. Values are presented in Table 2.

We observed significantly lower mean gestational age and mean birth weight in neonates of group A when compared to neonates of group 2 (Table 3).

Additionally, we observed that mean values of cord blood CRP were significantly higher $(p<0.05)$ in group $A$ when compared to group $B$, whereas there was not much change in total leucocyte count between the two groups.

Table 1: Mean \pm SD and statistical significance of parameters studied in neonates born to mothers with anemia and healthy controls

\begin{tabular}{lcccc}
\hline & Total $(N=60)$ & Group 1 $(N=30)$ & Group 2 $(N=30)$ & $\begin{array}{l}\text { Group 1 vs group 2 } \\
\text { Pvalue }\end{array}$ \\
\hline Parameters & Mean $\pm S D$ & Mean $\pm S D$ & $4.54 \pm 1.06$ & 0.02 \\
NPI (\%) & $3.69 \pm 1.2$ & $2.83 \pm 1.04$ & $12.94 \pm 1.15$ & 0.01 \\
Maternal blood Hb (g/L) & $11.16 \pm 2.51$ & $9.38 \pm 0.691$ & $37.93 \pm 5.2$ & 0.01 \\
Gestational age (weeks) & $32.50 \pm 7.68$ & $28.07 \pm 2.39$ & $3003.10 \pm 661.6$ & 0.00 \\
Birth weight (g) & $2679.25 \pm 457.9$ & $2355.40 \pm 385.65$ & & \\
\hline
\end{tabular}

Student's $t$-test and $p$ values; $p<0.05$ was considered as significant

Table 2: Mean \pm SD and statistical significance of parameters studied in neonates born to mothers with risk of EOS and healthy controls

\begin{tabular}{lccl}
\hline & $\begin{array}{c}\text { Group } A(N=61) \\
\text { Mean } \pm S D\end{array}$ & $\begin{array}{l}\text { Group } B(N=48) \\
\text { Mean } \pm S D\end{array}$ & $\begin{array}{l}\text { Group A vs group B } \\
\text { pvalue }\end{array}$ \\
\hline NPI (\%) & $2.94 \pm 1.10$ & $4.20 \pm 0.97$ & 0.0001 \\
CRP $(\mathrm{mg} / \mathrm{dL})$ & $49.13 \pm 42.72$ & $5.63 \pm 2.73$ & 0.0001 \\
Total leukocyte count & $17049.18 \pm 5256.00$ & $15485.42 \pm 5335.51$ & 0.12 \\
\hline
\end{tabular}

Unpaired $t$-test and $p$ values; $p<0.05$ was considered as significant

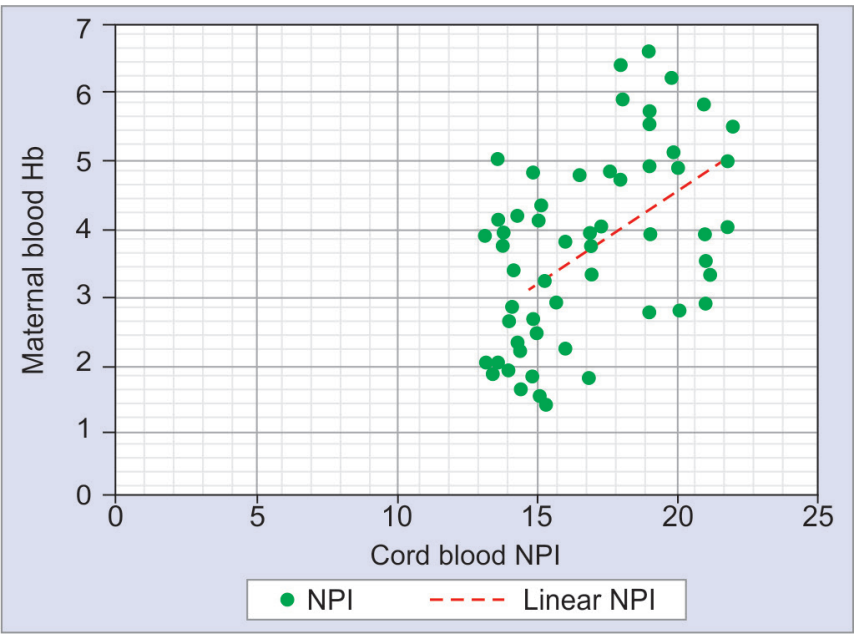

Fig. 1: Correlation of maternal $\mathrm{Hb}$ versus cord NPI

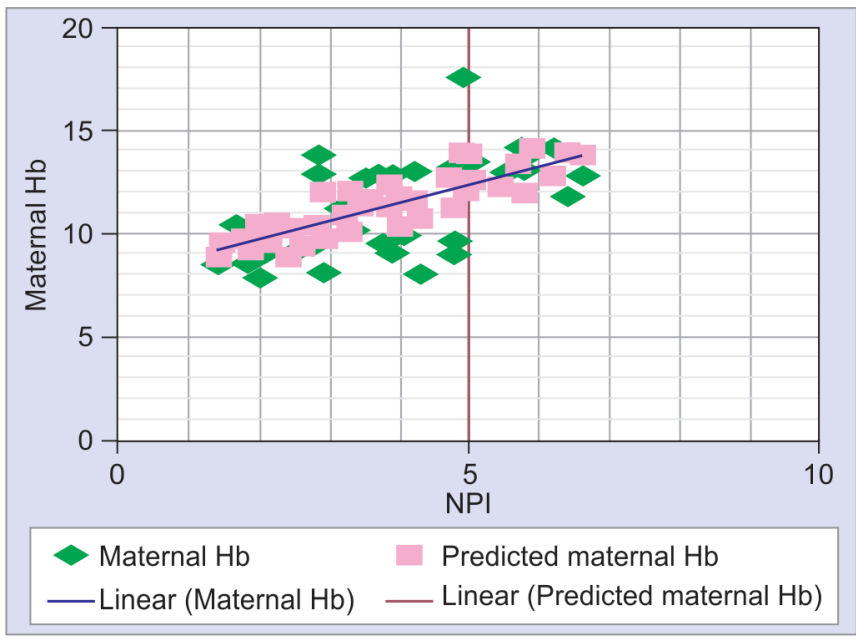

Fig. 2: Line fit plot showing a positive linear relationship between maternal $\mathrm{Hb}$ and $\mathrm{NPI}$ 
Table 3: Maternal, pregnancy, and neonatal characteristics of volunteers

\begin{tabular}{lllll}
\hline & & $\begin{array}{l}\text { Group } A \\
(N=61)\end{array}$ & $\begin{array}{l}\text { Group } B \\
(N=48)\end{array}$ & p value \\
\hline Gestational age & & $29.55 \pm 2.35$ & $38.13 \pm 4.1$ & 0.001 \\
Birth weight & & $1322.02 \pm 245.94$ & $3023.10 \pm 667.4$ & 0.0002 \\
Prolonged rupture of membrane & & 40 of 61 & - & - \\
Gender & M & 29 & 23 & 1.0 \\
& F & 32 & 25 & \\
Preeclampsia & Yes & 31 & - & \\
& No & 30 & - & \\
\hline
\end{tabular}

Values are expressed as mean with SD; unpaired $t$-test was used for analysis; $p<0.05$ was considered as significant

\section{Discussion}

The present study demonstrates the significance of evaluating NPI in two groups of neonates.

- Significantly lower levels of NPI are observed in cord blood of neonates born to anemic mothers, and an inverse relationship between maternal anemia and cord blood NPI highlights the impact of maternal iron status on proper functions of neutrophils.

- Significantly lower levels of NPI in cord blood of infants who are born with risk of EOS highlight the possibility of compromised innate immune functions in these neonates.

Normal levels of NPI indicate the efficiency of an innate immune response, with which neonates fight infections during early life. ${ }^{18,19}$ Decrease in NPI activity demonstrates the diminished capacity of the phagocytic neutrophils to kill the invading bacteria with potent microbicidal systems. ${ }^{20}$

Maternal iron supplementation, alone or in combination with other micronutrients, is said to improve infant and maternal health ${ }^{21}$ and immune status of the neonates dependent on the placental transfer of these nutrients. ${ }^{18,19}$

Reduced NPI in neonates born to mothers with anemia indicates compromised innate immune functions of the neutrophils and alarms that these neonates should be observed for any development of clinical sepsis soon after birth and should be treated early to prevent complications.

Decreased levels of NPI activity in cord blood neutrophils of neonates born with risk of EOS indicate underlying impairment of innate immune responses in these infants. Innate immunity is of great importance in early life before the infant develops complete immune competence. ${ }^{4}$ Thus, decreased NPI clearly suggests impeding danger for the neonate.

We found no studies to date that evaluated umbilical cord blood $\mathrm{NPI}$ as early immune markers.

However, reduced cord blood neutrophil chemotaxis and inherent defects in neutrophil migration (in response to bacterial products) have been reported in preterm infants. ${ }^{2,20}$ Prenatal environment has long been considered to impact general health outcomes and the development of the fetus. Earlier reports demonstrate endotoxin hyporesponsiveness seen in preterm fetus exposed to inflammation, as one factor for risk of EOS in neonates who are born preterm. It is well understood that intrauterine infection rather than the intrapartum acquisition of pathogens is one of the important causes of EOS. Therefore, cord blood serves as a source for biomarkers for fetal development. ${ }^{6,22}$ Decreased
NPI activity in cord blood neutrophils may be either a cause of or response to intrapartum infection. ${ }^{11,12}$

Phagocytic ability of neutrophils in newborn infants, a conserved process critical for cellular immunity, is reflected by NPI activity in cord blood. ${ }^{10}$

We also observed a significant increase in CRP levels in the cord blood of neonates with risk of EOS. This observation supports recent studies that focused on the elevation of CRP in the cord blood of neonates to predict EOS in preterm infants with intrauterine infections. A rise in CRP along with downstream of interleukin 6 suggests the occurrence of inflammatory response in EOS. ${ }^{23}$

Since the association between immune suppression of the fetus and inflammation is well established, we assume that the lowered NPI observed in our study is a result of several inflammatory processes in infants born with risk of EOS. Cytokine as an inflammatory marker is known to be increased in the cord blood of neonates born to mothers with prolonged premature rupture of membranes. ${ }^{24,25}$

The observations of our study provide an important foundation for newer approaches using cord blood markers as diagnostic indicators of EOS. Since umbilical cord blood obtained at delivery constitutes a noninvasive sampling approach, new useful immune biomarkers and biomarkers for early sepsis diagnosis, measurable in the cord blood, will be very interesting from a clinical point of view.

\section{Limitations of the Study}

However, our study is limited by the relatively small sample size, and only NPI being evaluated as an immune marker, nevertheless, it opens the possibility for further studies on neutrophil phagocytic capacity, which may enable us to understand more clearly about the involvement of these first-line defenders in EOS.

\section{Recommendations}

However, these findings need to be verified by prospective multicenter studies and interpretation of these biomarkers for EOS with follow-up studies.

Further studies on cord blood inflammatory and immune markers may lead to improved diagnosis and early treatment strategies that can be employed to effectively treat infants born with risk factors of developing EOS.

\section{Conclusion}

Decreased cord blood NPI may mark early neonatal immune deficiency in infants born to anemic mothers and in infants born with risk of developing EOS. 
Identification of NPI in the cord blood will help to identify neonatal immune dysfunctions that may lead to EOS. Reduced NPI in the neonates born to mothers emphasizes the need for maternal iron supplements to reduce the risk of low birth weight, premature birth, and EOS and justifies the need for micronutrient interventions with antenatal care.

Further studies on cord blood NPI will lead to identifying NPI as an early predictor of EOS, which will help to improve diagnostics and early therapeutic intervention.

\section{References}

1. Moreno-Fernandez J, Ochoa JJ, Latunde-Dada GO, et al. Iron deficiency and iron homeostasis in low birth weight preterm infants: a systematic review. Nutrients 2019;11(5):1090. DOI: 10.3390/nu11051090.

2. Raymond SL, Mathias BJ, Murphy TJ, et al. Neutrophil chemotaxis and transcriptomics in term and preterm neonates. Transl Res 2017;190:4-15. DOI: 10.1016/j.trsl.2017.08.003.

3. Mukhopadhyay S, Puopolo KM. Neonatal early-onset sepsis: epidemiology and risk assessment. NeoReviews 2015;16(4):e221e230. DOI: 10.1542/neo.16-4-e221.

4. Jennewein MF, Abu-Raya B, Jiang $Y$, et al. Transfer of maternal immunity and programming of the newborn immune system. Semin Immunopathol 2017;39(6):605-613. DOI: 10.1007/s00281-017-0653-x.

5. Oddie S. Risk factors for early onset neonatal group B streptococcal sepsis: case-control study. BMJ 2002;325(7359):308. DOI: 10.1136/ bmj.325.7359.308.

6. Cunningham-Rundles $\mathrm{S}$, Lin $\mathrm{H}, \mathrm{Ho}$-Lin $\mathrm{D}$, et al. Role of nutrients in the development of neonatal immune response. Nutr Rev 2009b;67(Suppl. 2):S152-S163. DOI: 10.1111/j.1753-4887.2009.00236.x.

7. Bashir M. Pregnancy outcome in anemic pregnant women: impact of dietary intervention. Int J Nurs Midwifery Res 2018;05(01):37-45. DOI: 10.24321/2455.9318.201809.

8. Lee HS, Kim MS, Kim MH, et al. Iron status and its association with pregnancy outcome in Korean pregnant women. Eur J Clin Nutr 2006;60(9):1130-1135. DOI: 10.1038/sj.ejcn.1602429.

9. El-Farrash RA, Ismail EAR, Nada AS. Cord blood iron profile and breast milk micronutrients in maternal iron deficiency anemia. Pediatr Blood Cancer 2011;58(2):233-238. DOI: 10.1002/pbc.23184.

10. Wynn JL, Wong HR. Pathophysiology and treatment of septic shock in neonates. Clin Perinatol 2010;37(2):439-479. DOI: 10.1016/j. clp.2010.04.002.

11. Cuenca A, Wynn J, Moldawer L, et al. Role of innate immunity in neonatal infection. Am J Perinatol 2013;30(02):105-112. DOI: 10.1055/ s-0032-1333412.
12. Gomez-Lopez N, Romero R, Xu Y, et al. Are amniotic fluid neutrophils in women with intraamniotic infection and/or inflammation of fetal or maternal origin? Am J Obstet Gynecol 2017;217(6):693.e1-693.e16. DOI: 10.1016/j.ajog.2017.09.013.

13. Greenberg $S$, Grinstein S. Phagocytosis and innate immunity. Curr Opin Immunol 2002;14(1):136-145. DOI: 10.1016/s0952-7915(01)00309-0.

14. Rosales $C$. Neutrophils at the crossroads of innate and adaptive immunity. J Leukocyte Biol 2020;108(1):377-396. DOI: 10.1002/ JLB.4MIR0220-574RR.

15. Jutras I, Desjardins M. Phagocytosis: at the crossroads of innate and adaptive immunity. Ann Rev Cell Developmental Biol 2005;21(1):511527. DOI: 10.1146/annurev.cellbio.20.010403.102755.

16. Nunes P, Demaurex N, Dinauer MC. Regulation of the NADPH oxidase and associated ion fluxes during phagocytosis. Traffic 2013;14(11):1118-1131. DOI: 10.1111/tra.12115.

17. Muniz-Junqueira MI, Peçanha LMF, da Silva-Filho VL, et al. Novel microtechnique for assessment of postnatal maturation of the phagocytic function of neutrophils and monocytes. Clin Diagn Lab Immunol 2003;10(6):1096-1102. DOI: 10.1128/cdli.10.6.1096-1102.2003.

18. Azizia M, Lloyd J, Allen M, et al. Immune status in very preterm neonates. Pediatrics 2012;129(4):e967-e974. DOI: 10.1542/peds.20111579.

19. Devarshi PP, Grant RW, Ikonte CJ, et al. Maternal omega-3 nutrition, placental transfer and fetal brain development in gestational diabetes and preeclampsia. Nutrients 2019;11(5):1107. DOI: 10.3390/ nu11051107.

20. Birle A, Nebe CT, Hill S, et al. Neutrophil chemotaxis in cord blood of term and preterm neonates is reduced in preterm neonates and influenced by the mode of delivery and anaesthesia. PLoS One 2015;10(4):e0120341. DOI: 10.1371/journal.pone.0120341.

21. Gernand AD, Schulze KJ, Stewart CP, et al. Micronutrient deficiencies in pregnancy worldwide: health effects and prevention. Nat Rev Endocrinol 2016;12(5):274-289. DOI: 10.1038/nrendo.2016.37.

22. Reddy R, Kher A. Outcome of neonates born to mothers with premature rupture of membranes. Sri Lanka J Child Health 2020;49(3):256-262. DOI: 10.4038/sljch.v49i3.9144.

23. Mithal LB, Palac HL, Yogev R, et al. Cord blood acute phase reactants predict early onset neonatal sepsis in preterm infants. PLoS One 2017;12(1):e0168677. DOI: 10.1371/journal.pone.0168677.

24. Raghupathy R. Cytokine patterns in maternal blood after premature rupture of membranes. Obstet Gynecol 2001;98(1):122-126. DOI: 10.1016/s0029-7844(01)01408-9.

25. Wolfs TGAM, Jellema RK, Turrisi G, et al.Inflammation-induced immune suppression of the fetus: a potential link between chorioamnionitis and postnatal early onset sepsis. J Matern Fetal Neonatal Med 2012;25(Suppl. 1):8-11. DOI: 10.3109/14767058.2012.664447. 